COMMUNICATIONS IN ALGEBRA ${ }^{\circledR}$

Vol. 31, No. 10, pp. 4745-4763, 2003

\title{
The 2-Power Degree Subfields of the Splitting Fields of Polynomials with Frobenius Galois Groups
}

\author{
Blair K. Spearman, ${ }^{1}$ Kenneth S. Williams, ${ }^{2, *}$ \\ and Qiduan Yang ${ }^{1}$ \\ ${ }^{1}$ Department of Mathematics and Statistics, Okanagan University \\ College, Kelowna, British Columbia, Canada \\ ${ }^{2}$ School of Mathematics and Statistics, Carleton University, Ottawa, \\ Ontario, Canada
}

\begin{abstract}
Let $f(x)$ be an irreducible polynomial of odd degree $n>1$ whose Galois group is a Frobenius group. We suppose that the Frobenius complement is a cyclic group of even order $h$. Let $2^{t} h$. For each $i=1,2, \ldots, t$ we show that the splitting field $L$ of $f(x)$ has exactly one subfield $K_{i}$ with $\left[K_{i}: \mathbb{Q}\right]=2^{i}$. These subfields form a tower of normal extensions $\mathbb{Q} \subset K_{1} \subset K_{2} \subset \cdots \subset K_{t}$ with $\left[K_{i}: K_{i-1}\right]=2(i=1,2, \ldots, t)$ and $K_{0}=\mathbb{Q}$. Our main result in this paper is an explicit formula for an element $\alpha_{i}$ in $K_{i-1}$ such that $K_{i}=\mathbb{Q}\left(\sqrt{\alpha_{i}}\right)(i=1,2, \ldots, t)$. This result is applied to DeMoivre's quintic $x^{5}-5 a x^{3}+5 a^{2} x-b$, solvable
\end{abstract}

*Correspondence: Kenneth S. Williams, School of Mathematics and Statistics, Carleton University, Ottawa, Ontario K1S 5B6, Canada; E-mail: williams@, math.carleton.ca.

4745

DOI: $10.1081 /$ AGB-120023130

0092-7872 (Print); 1532-4125 (Online)

Copyright (C) 2003 by Marcel Dekker, Inc.

www.dekker.com 
quintic trinomials $x^{5}+a x+b$, as well as to some numerical polynomials of degrees 5,9 , and 13 .

Key Words: Frobenius group; Subfields of splitting field; Galois group.

\section{INTRODUCTION}

A finite group $G$ is said to be a Frobenius group if there exists a transitive $G$-set $X$ such that

every $g \in G \backslash\{1\}$ has at most one fixed point

and

there is some $g \in G \backslash\{1\}$ that does not have a fixed point.

It can be proved (Rotman, 2002, Proposition 8.161) that a finite group $G$ is a Frobenius group if and only if it contains a proper nontrivial subgroup $H$ such that

$$
H \cap g H g^{-1}=\{1\} \quad \text { for all } g \notin H .
$$

Such a subgroup $H$ of $G$ is called a Frobenius complement of $G$. Let

$$
N=\{1\} \cup\left(G \backslash\left(\bigcup_{g \in G} g H g^{-1}\right)\right) .
$$

$N$ is called the Frobenius kernel of $G$. Frobenius proved using character theory the following result (Rotman, 2002, Theorem 8.164):

Let $G$ be a Frobenius group with complement $H$ and kernel $N$.

Then $N$ is a normal subgroup of $G$ with $N \cap H=\{1\}$ and $G=N H$.

Furthermore, we have (Robinson, 1982, Ex. 8.5.6)

$h \mid n-1, \quad$ where $h=|H|$ and $n=|N|$.

By (4), $G$ is the semi-direct product of $N$ and $H$, written $G=N \rtimes H$. Note that there is a natural $G$-action on $N$ : for $\sigma$ in $G, \phi_{\sigma}(v)=\sigma v \sigma^{-1}, \nu \in N$. We state the following result without proof. 
The semi-direct product $G=N \rtimes H$ is a Frobenius group with kernel $N$ and complement $H$ if and only if the action of $H \backslash\{1\}$ on $N \backslash\{1\}$ is fixed-point free, that is, if $\sigma \in H, v \in N \backslash\{1\}$ and $\sigma v \sigma^{-1}=v$ imply $\sigma=1$.

In this paper, we consider irreducible polynomials $f(x) \in \mathbb{Z}[x]$ with Galois group $G=\operatorname{Gal}(f)$ satisfying the following three conditions:

$G=N \rtimes H$ is a Frobenius group with kernel $N$ and complement $H$,

$H$ is a cyclic group with even degree $h$, hence $N$ is abelian,

$\operatorname{deg}(f(x))$ is odd, greater than 1 , and equal to $n$, the order of $N$.

In (7b) the fact that $N$ is abelian follows from Robinson (1982, Ex. 10.5). We define the positive integer $t$ by

$$
2^{t} \| h,
$$

and the odd positive integer $h_{1}$ by

$$
h_{1}=h / 2^{t} .
$$

We denote the splitting field of $f(x)$ by $L$ so that

$$
\operatorname{Gal}(L / \mathbb{Q})=\operatorname{Gal}(f)=G=N \rtimes H .
$$

For each $j=1,2, \ldots, t$ we show that $L$ has exactly one subfield $K_{j}$ with $\left[K_{j}: \mathbb{Q}\right]=2^{j}$. These subfields form a tower of normal extensions $\mathbb{Q} \subset K_{1} \subset K_{2} \subset \cdots \subset K_{t}$ with $\left[K_{i}: K_{i-1}\right]=2(i=1,2, \ldots, t)$ where $K_{0}=\mathbb{Q}$. Our objective in this paper is to give an explicit element $\alpha_{i} \in K_{i-1}$ such that $K_{i}=\mathbb{Q}\left(\sqrt{\alpha_{i}}\right)(i=1,2, \ldots, t)$. This determination is given in Sec. 3 after some preliminary results are proved in Sec. 2. In Sec. 4 we apply our results to certain classes of polynomials.

Remark 1. Let $K$ be a subfield of $\mathbb{C}$. Let $\theta_{1}, \theta_{2}, \ldots, \theta_{n}$ be the roots in $\mathbb{C}$ of $f(x) \in K[x]$. The discriminant of $f(x)$ is defined by

$$
D_{f}=\prod_{\substack{i, j=1 \\ i<j}}^{n}\left(\theta_{i}-\theta_{j}\right)^{2} .
$$


If the roots of $f(x)$ are distinct, we fix some ordering of the roots and view the Galois group $G$ of $f(x)$ as a subgroup of the symmetric group $S_{n}$. Galois theory tells us that the field $K\left(\sqrt{D_{f}}\right)$ is always a subfield of the splitting field of $f(x)$, and that $G$ is a subgroup of the alternating group $A_{n}$ if and only if $\sqrt{D_{f}} \in K$. Therefore the field extension $K\left(\sqrt{D_{f}}\right) / K$ is quadratic if and only if $G$ contains odd permutations on $\left\{\theta_{1}, \theta_{2}, \ldots\right.$, $\left.\theta_{n}\right\}$. In this paper, we shall see that when $G$ is not contained in $A_{n}$, the quadratic extension $K_{1} / K$ is reproducing $K\left(\sqrt{D_{f}}\right) / K$. It is worth noting that even when $G$ is not a subgroup of $A_{n}$, a quadratic tower over $K$ can still be constructed.

Definition 1. Let $\theta_{1}, \theta_{2}, \ldots, \theta_{n}$ be the roots in $\mathbb{C}$ of $f(x) \in K[x]$. The discriminant polynomial of $f(x)$ is defined to be

$$
g(x)=\prod_{\substack{i, j=1 \\ i \neq j}}^{n}\left(x-\left(\theta_{i}-\theta_{j}\right)\right) .
$$

It is clear that $g(x) \in K[x]$ and $\operatorname{deg} g(x)=n(n-1)$.

We now state our main result.

Theorem. Let $f(x) \in \mathbb{Z}[x]$ be an irreducible polynomial. Let the roots of $f(x)$ in $\mathbb{C}$ be $\theta_{1}, \theta_{2}, \ldots, \theta_{n}$. Let $L=\mathbb{Q}\left(\theta_{1}, \theta_{2}, \ldots, \theta_{n}\right)$ be the splitting field of $f(x)$, and $G=\operatorname{Gal}(f)=\operatorname{Gal}(L / \mathbb{Q})$ be the Galois group of $f(x)$. Assume that $f(x)$ and $G$ satisfy the following four conditions:

(a) $G=N \rtimes H$ is a Frobenius group with kernel $N$ and complement $H$.

(b) $H$ is a cyclic group with even degree $h$.

(c) $\operatorname{deg}(f(x))$ is odd, greater than 1, and equal to $n$ the order of $N$.

(d) The discriminant polynomial of $f(x)$ is squarefree.

Define $t$ and $h_{1}$ as in (8) and (9) respectively. Then L contains exactly one normal subfield $K_{j}$ with $\left[K_{j}: \mathbb{Q}\right]=2^{j}$ for each $j=1,2, \ldots, t$. These subfields satisfy

$$
\mathbb{Q} \subset K_{1} \subset K_{2} \subset \cdots \subset K_{t}
$$

with $K_{i} / \mathbb{Q}$ a cyclic extension of degree $2^{i}$ for $i=0,1, \ldots, t$. Further, for $i=0,1, \ldots, t-1$,

$$
g(x)=\prod_{j=1}^{2^{i}(n-1) / h} g_{i j}(x),
$$


where each $g_{i j}(x) \in K_{i}[x]$ is monic, irreducible, of degree $n h / 2^{i}$, and even. Finally, for any $j \in\left\{1,2, \ldots, 2^{i}(n-1) / h\right\}$, we have

$$
K_{i+1}=\mathbb{Q}\left(\sqrt{g_{i j}(0)}\right), \quad \text { for } i=0,1,2, \ldots, t-2,
$$

and

$$
K_{t}=\mathbb{Q}\left(\sqrt{-g_{t-1 j}(0)}\right) .
$$

Remark 2. The existence of a quadratic tower of the form (11) follows from Galois theory. Let $L^{N}$ be the subfield of $L$ fixed by $N$. Then the Galois group of $L^{N}$ over $\mathbb{Q}, \operatorname{Gal}\left(L^{N} / \mathbb{Q}\right)$, is isomorphic to $G / N$, hence to $H$, which is cyclic of order $2^{t} h_{1}$. $\operatorname{Gal}\left(L^{N} / \mathbb{Q}\right)$ has a unique sequence of subgroups (each of which is normal since $\operatorname{Gal}\left(L^{N} / \mathbb{Q}\right)$ is abelian)

$$
P_{t} \triangleleft P_{t-1} \triangleleft \cdots \triangleleft P_{1} \triangleleft P_{0}=\operatorname{Gal}\left(L^{N} / \mathbb{Q}\right),
$$

such that $\left[P_{i-1}: P_{i}\right]=2, i \in\{1,2, \ldots, t\}$. Correspondingly, $G=\operatorname{Gal}\left(L^{N} / \mathbb{Q}\right)$ has a unique sequence of normal subgroups

$$
M_{t} \triangleleft M_{t-1} \triangleleft \cdots M_{1} \triangleleft M_{0}=G,
$$

such that $\left[M_{i-1}: M_{i}\right]=2, i \in\{1,2, \ldots, t\}$, and $N \subseteq M_{i}, i \in\{0,1, \ldots, t\}$, by the Correspondence Theorem (Rotman, 2002, Proposition 2.76). A quadratic tower of the form (11) thus exists in which each $K_{i}$ is the fixed field of $M_{i}$ for $i \in\{1,2, \ldots, t\}$. Moreover, we claim that every subfield of $L$ of degree $2^{j}$ over $\mathbb{Q}$ must be a field in this tower. Such a subfield, written as $L^{M}$, is fixed by a subgroup $M$ of $G$ such that $[G: M]=2^{j}, j \in\{1$, $2, \ldots, t\}$. We notice that $\frac{|M N|}{|M|}$ is a power of 2 , as it is a factor of $[G: M]$. On the other hand $\frac{|M N|}{|M|}=\frac{|N|}{|M \cap N|}$ is odd since $|N|$ is odd. Hence $\frac{|M N|}{|M|}=1$. This shows that $N \subseteq M$. Therefore $M$ must be the subgroup $M_{j}$ in (15) and it follows that the subfield $L^{M}$ is the field $K_{j}$ in (11). This implies the uniqueness of the tower (11). The following diagram illustrates the Galois correspondence between some subgroups of $G$ and some subfields of $L$.

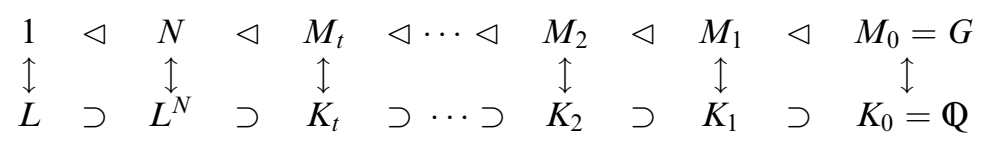




\section{SOME PRELIMINARY RESULTS}

We recall and reorganize some basic facts about Frobenius groups in Cangelmi 2000 and Robinson (1982) for our purposes. Let $f(x) \in \mathbb{Z}[x]$ satisfy all the assumptions of the Theorem. Let $\left\{\theta_{1}, \theta_{2}, \ldots, \theta_{n}\right\}$ be the roots of $f(x)$ in $\mathbb{C}$. We may replace $\mathbb{Q}$ by a number field $K$. For a fixed $i \in\{1,2, \ldots, n\}$, let $H_{i}$ be the stabilizer of $\theta_{i}$ in $G$, that is, $H_{i}=\left\{\sigma \in G: \sigma\left(\theta_{i}\right)=\theta_{i}\right\}$. Then the subfield of the splitting field $L$ fixed by $H_{i}$ is $K\left(\theta_{i}\right)$. As $f(x)$ is irreducible over $K$, we have

$$
\left[G: H_{i}\right]=\left[K\left(\theta_{i}\right): K\right]=\operatorname{deg}(f(x))=|N|=[G: H] .
$$

It follows that $\left|H_{i}\right|=|H|$, hence $N \cap H_{i}=\{1\}, i=1,2, \ldots, n$, since $\left|H_{i}\right|=h$ and $|N|=n$ are coprime by (5). The natural projection $\sigma \in G \rightarrow \sigma N$ restricted to the subgroup $H_{i}$ must be one-to-one because the kernel of the map is $N \cap H_{i}=\{1\}$. Therefore $H_{i} \cong G / N \cong H$ as groups. As $G$ is transitive on the set $\left\{\theta_{1}, \theta_{2}, \ldots, \theta_{n}\right\}$, for any $j \in\{1,2, \ldots, n\}$ with $j \neq i$ there exists $g \in G$ such that $g\left(\theta_{i}\right)=\theta_{j}$. Then the group $g H_{i} g^{-1}$ (a conjugate of $\left.H_{i}\right)$ is the stabilizer $H_{j}$ of the root $\theta_{j}$. Thus $H_{i}$ has exactly $n$ conjugates including itself, and each of these fixes exactly one root of $f(x)$. The stabilizer of two distinct roots of $f(x)$ is the trivial subgroup $\{1\}$ of $G$, since $H_{i} \cap H_{j}=\{1\}$ for $i \neq j$. It is clear that (3) is satisfied and $G$ is a Frobenius group with complement $H_{i}$ for any $i \in\{1,2, \ldots, n\}$. From the orders of $N, H_{i}$ and $G$, it is not hard to verify that

$$
N=\{1\} \cup\left(G \backslash\left(\bigcup_{g \in G} g H g^{-1}\right)\right) .
$$

Thus $N$ is the Frobenius kernel with respect to the complement $H_{i}$ of $G$. The following is a summary of the above discussion.

Lemma 1. Let $G=N \rtimes H$ be a Frobenius group serving as the Galois group of an irreducible polynomial $f(x)$ over a number field $K$, such that $\operatorname{deg}(f(x))=n=|N|$. Let $\left\{\theta_{1}, \theta_{2}, \ldots, \theta_{n}\right\}$ be the set of all roots of $f(x)$ in C. Then

(i) $G=N \rtimes H_{i}$, where $H_{i}=\left\{\sigma \in G: \sigma\left(\theta_{i}\right)=\theta_{i}\right\}, i \in\{1,2, \ldots, n\}$.

(ii) The set $N \backslash\{1\}$ contains all elements in $G$ that do not have a fixed point in $\left\{\theta_{1}, \theta_{2}, \ldots, \theta_{n}\right\}$.

(iii) If $\sigma \in G$ and $\sigma\left(\theta_{r}\right)=\theta_{r}, \sigma\left(\theta_{s}\right)=\theta_{s}$ for $r, s \in\{1,2, \ldots, n\}$ with $r \neq s$, then $\sigma=1$.

The following result is an easy corollary of Lemma 1. 
Proposition 1. Keep the assumptions in Lemma 1. Let $i$ be a fixed integer in $\{1,2, \ldots, n\}$. If $H$ is a cyclic group then there exists $\alpha \in G$ such that $G=N \rtimes\langle\alpha\rangle$ and $\alpha\left(\theta_{i}\right)=\theta_{i}$.

Proof. The subgroup $H_{i}$ is cyclic since $H_{i} \cong H$. Let $\alpha$ be a generator of $H_{i}$ and the statement follows.

Now we turn to some properties of the Frobenius kernel $N$.

Proposition 2. For any $i \in\{1,2, \ldots, n\}, N$ is a complete set of left coset representatives of $H_{i}$ in $G$.

Proof. Assume that $\nu_{1} \in N, \nu_{2} \in N$ and $\nu_{1} H_{i}=\nu_{2} H_{i}$ so that $\nu_{1}^{-1} \nu_{2} \in H_{i}$. Hence $\nu_{1}^{-1} \nu_{2}=1$ since $N \cap H_{i}=\{1\}$. Thus $\nu_{1}=\nu_{2}$. The proposition now follows from the fact $|N|=\left[G: H_{i}\right]$.

Proposition 3. The Frobenius kernel $N$ acts transitively on the set of roots $\left\{\theta_{1}, \theta_{2}, \ldots, \theta_{n}\right\}$ of $f(x)$.

Proof. For $r, s \in\{1,2, \ldots, n\}, r \neq s$, there exists $\sigma \in G$, such that $\sigma\left(\theta_{r}\right)=\theta_{s}$, since $G$ acts transitively on the set $\left\{\theta_{1}, \theta_{2}, \ldots, \theta_{n}\right\}$. By Proposition 2, $\sigma \in \nu H_{r}$ for some $\nu \in N$. Thus $\sigma=\nu \eta$ for some $\eta \in H_{r}$. Now we have

$$
\nu\left(\theta_{r}\right)=\nu \eta\left(\theta_{r}\right)=\sigma\left(\theta_{r}\right)=\theta_{s}
$$

completing the proof.

Next we consider the subgroups of $G$ of the form $N \rtimes\left\langle\alpha^{2^{m}}\right\rangle$, $m \in\{0,1,2, \ldots, t\}$.

Proposition 4. For $m \in\{0,1,2, \ldots, t\}$, we have

(i) $N \rtimes\left\langle\alpha^{2^{m}}\right\rangle$ is a subgroup of $G$ containing $N$.

(ii) The index of $N \rtimes\left\langle\alpha^{2^{m}}\right\rangle$ in $G$ is $2^{m}$.

(iii) $N \rtimes\left\langle\alpha^{2^{m}}\right\rangle$ acts transitively on $\left\{\theta_{1}, \theta_{2}, \ldots, \theta_{n}\right\}$, the set of roots of $f(x)$.

(iv) $N \rtimes\left\langle\alpha^{2^{m}}\right\rangle$ is a Frobenius group with Frobenius kernel $N$ and complement $\left\langle\alpha^{2^{m}}\right\rangle$.

Proof. (i) is obvious. (ii) follows from the calculations

$$
\left[N \rtimes\langle\alpha\rangle: N \rtimes\left\langle\alpha^{2^{m}}\right\rangle\right]=\frac{|N||\alpha|}{|N|\left|\alpha^{2^{m}}\right|}=\frac{h}{h / 2^{m}}=2^{m} .
$$


To prove (iii) we notice that, by Proposition $3, N$ acts transitively on the set $\left\{\theta_{1}, \theta_{2}, \ldots, \theta_{n}\right\}$. So does $N \rtimes\left\langle\alpha^{2^{m}}\right\rangle$.

Now conditions (1) and (2) in Sec. 1 are satisfied when $\left\{\theta_{1}, \theta_{2}, \ldots, \theta_{n}\right\}$ is considered as the $N \rtimes\left\langle\alpha^{2^{m}}\right\rangle$-set. This proves (iv).

In Remark 2, we observed that $G=\operatorname{Gal}(L / \mathbb{Q})$ has a unique sequence of normal subgroups

$$
M_{t} \triangleleft M_{t-1} \triangleleft \cdots M_{1} \triangleleft M_{0}=G,
$$

such that $\left[M_{i-1}: M_{i}\right]=2, i \in\{1,2, \ldots, t\}$, and $N \subseteq M_{i}, i \in\{0,1,2, \ldots, t\}$. Combining this observation and Proposition 4 , we obtain

\section{Proposition 5.}

(i) $M_{m}=N \rtimes\left\langle\alpha^{2^{m}}\right\rangle, m \in\{0,1,2, \ldots, t\}$.

(ii) $K_{m}$ is the subfield of $L$ fixed by $M_{m}=N \rtimes\left\langle\alpha^{2^{m}}\right\rangle, \quad m \in$ $\{0,1,2, \ldots, t\}$.

Proposition 6. For $r, s \in\{1,2, \ldots, n\}$ with $r \neq s$, there exists $\tau \in G$ such that $\tau\left(\theta_{r}\right)=\theta_{s}$ and $\tau\left(\theta_{s}\right)=\theta_{r}$.

Proof. For any $i \in\{1,2, \ldots, n\}$ the subgroup $H_{i}=\left\{\sigma \in G: \sigma\left(\theta_{i}\right)=\theta_{i}\right\}$ is cyclic of even order. Denote the unique element of order 2 in $H_{i}$ by $\tau_{i}$. If $\tau \in G$ is of order 2, then $\tau$ lies in $H_{i}$ for some $i \in\{1,2, \ldots, n\}$, since $G=$ $\left(\bigcup_{i=1}^{n} H_{i}\right) \cup N$ and $|N|$ is odd. Thus $\tau=\tau_{i}$ for some $i$ and $\left\{\tau_{1}, \tau_{2}, \ldots, \tau_{n}\right\}$ is the complete set of order 2 elements in $G$. Each $\tau_{i}(i \in\{1,2, \ldots, n\})$ fixes exactly one root $\theta_{i}$ of $f(x)$, hence $\tau_{i}$ is a product of $(n-1) / 2$ transpositions. We point out that no two of these order 2 elements can have a transposition in common. Otherwise, say that the transposition $\left(\theta_{r}, \theta_{s}\right)$, for some $r \neq s$, occurs in both $\tau_{i}$ and $\tau_{j}$, for some $i \neq j$. Then

$$
\begin{aligned}
& \tau_{i} \tau_{j}\left(\theta_{r}\right)=\tau_{i}\left(\theta_{s}\right)=\theta_{r}, \\
& \tau_{i} \tau_{j}\left(\theta_{s}\right)=\tau_{i}\left(\theta_{r}\right)=\theta_{s} .
\end{aligned}
$$

It follows from Lemma 1 (iii) that $\tau_{i} \tau_{j}=1$, hence $\tau_{i}=\tau_{j}$, a contradiction. Now assume that $r, s \in\{1,2, \ldots, n\}$ and $r \neq s$. Then there are $(n-2)$ order 2 elements in $G$ which fix neither $\theta_{r}$ nor $\theta_{s}$. Let $\tau_{k}$ be such an order 2 element. Then $k \neq r$ and $k \neq s . \tau_{k}$ contains a transposition $\left(\theta_{r}, \tau_{k}\left(\theta_{r}\right)\right)$, where $\tau_{k}\left(\theta_{r}\right) \in\left\{\theta_{1}, \theta_{2}, \ldots, \theta_{n}\right\} \backslash\left\{\theta_{r}, \theta_{k}\right\}$, which is a set of $(n-2)$ elements containing $\theta_{s}$. Therefore there exists $\tau \in G$, such that $\tau\left(\theta_{r}\right)=\theta_{s}$ and $\tau\left(\theta_{s}\right)=\theta_{r}$.

In the rest of this section we assume the following set of conditions. 


\section{Condition Set.}

(i) $K$ is a subfield of $\mathbb{C}$ and $\theta_{1}, \theta_{2}, \ldots, \theta_{n}$ are the roots in $\mathbb{C}$ of an irreducible polynomial $f(x) \in K[x]$.

(ii) The discriminant polynomial of $f(x)$

$g(x)=\prod_{\substack{i, j=1 \\ i \neq j}}^{n}\left(x-\left(\theta_{i}-\theta_{j}\right)\right)$

is squarefree.

(iii) $L=K\left(\theta_{1}, \theta_{2}, \ldots, \theta_{n}\right)$ is the splitting field of $f(x)$.

(iv) $G^{*}=\operatorname{Gal}(L / K)$ is a Frobenius group with Frobenius kernel $N$ and complement $H^{*}$, such that $H^{*}$ is a cyclic group with order $\left|H^{*}\right|=2^{m} h_{1}$, where $m$ is a positive integer and $h_{1}$ is an odd positive integer.

(v) The degree of $f(x)$ is odd, greater than 1 , and equal to $n$, the order of $N$.

Let $\bar{g}(x)$ be an irreducible factor of $g(x)$ over $K$. We have the following observations.

Proposition 7. The group $G^{*}=\operatorname{Gal}(L / K)$ acts transitively on the set of roots of $\bar{g}(x)$. Moreover, $G^{*}$ acts regularly on the set of roots of $\bar{g}(x)$, that is, the stabilizer of any root of $\bar{g}(x)$ in $G^{*}$ is the trivial subgroup $\{1\}$.

Proof. The first statement is clear. A root of $\bar{g}(x)$ is of the form $\theta_{r}-\theta_{s}$, for some $r \neq s, r, s \in\{1,2, \ldots, n\}$. If $\sigma \in G^{*}$ and $\sigma\left(\theta_{r}-\theta_{s}\right)=\theta_{r}-\theta_{s}$, then $\sigma\left(\theta_{r}\right)=\theta_{r}$ and $\sigma\left(\theta_{s}\right)=\theta_{s}$, since $g(x)$ is squarefree. Thus $\sigma=1$ by Lemma 1(iii).

Corollary. The degree of $\bar{g}(x)$ is equal to $\left|G^{*}\right|$.

We note that the discriminant polynomial $g(x)$ is the polynomial $R(-1, f)(x)$ in Cangelmi $(2000$, p. 852). A more general treatment can be found in Cangelmi (2000, Theorem 3.1).

\section{Proposition 8.}

(i) If $\theta_{r}-\theta_{s}$ is a root of $\bar{g}(x)$, for some $r, s \in\{1,2, \ldots, n\}$ with $r \neq s$, so is $\theta_{s}-\theta_{r}$.

(ii) $\bar{g}(x)=h\left(x^{2}\right)$ for some $h(x) \in K[x]$. 
Proof. By Proposition 6, there exists $\tau \in G^{*}$, such that $\tau\left(\theta_{r}\right)=\theta_{s}$ and $\tau\left(\theta_{s}\right)=\theta_{r}$. Thus $\tau\left(\theta_{r}-\theta_{s}\right)=\theta_{s}-\theta_{r}$ is a root of $\bar{g}(x)$ if $\theta_{r}-\theta_{s}$ is a root of $\bar{g}(x)$. Over $L$, whenever $x-\left(\theta_{r}-\theta_{s}\right)$ is a linear factor of $\bar{g}(x)$, so is $x-\left(\theta_{s}-\theta_{r}\right)$. Therefore $\bar{g}(x)$ is a product of quadratic factors of the form $x^{2}-\left(\theta_{r}-\theta_{s}\right)^{2}$ for some $r, s \in\{1,2, \ldots, n\}$ with $r \neq s$. This proves (ii).

We note that $d=\left|G^{*}\right| / 2$ is the degree of $h(x)$. Next we label the roots $\xi_{1}, \ldots, \xi_{d}, \xi_{d+1}, \ldots, \xi_{2 d}$ of $\bar{g}(x)$ in such a way that $\xi_{k}=-\xi_{k+d}$, $k=1,2, \ldots, d$. We observe that

$$
\begin{aligned}
\bar{g}(x) & =\prod_{k=1}^{d}\left(x-\xi_{k}\right)\left(x+\xi_{k}\right)=\prod_{k=1}^{d}\left(x^{2}-\xi_{k}^{2}\right), \\
\bar{g}(0) & =(-1)^{d} \prod_{k=1}^{d} \xi_{k}^{2}, \\
h(x) & =\prod_{k=1}^{d}\left(x-\xi_{k}^{2}\right), \\
D_{h} & =\prod_{1 \leq k<l \leq d}\left(\xi_{k}^{2}-\xi_{l}^{2}\right)^{2} .
\end{aligned}
$$

Then we have

$$
\begin{aligned}
D_{\bar{g}} & =\prod_{1 \leq k<l \leq 2 d}\left(\xi_{k}-\xi_{l}\right)^{2} \\
& =\left[\prod_{1 \leq k<l \leq d}\left(\xi_{k}-\xi_{l}\right)^{2}\right]^{2}\left[\prod_{k=1}^{d}\left(2 \xi_{k}\right)^{2}\right]\left[\prod_{1 \leq k<l \leq d}\left(\xi_{k}+\xi_{l}\right)^{2}\right]^{2} \\
& =\left[\prod_{1 \leq k<l \leq d}\left(\xi_{k}^{2}-\xi_{l}^{2}\right)^{2}\right]^{2}\left(2^{2 d}\right) \prod_{k=1}^{d} \xi_{k}^{2} \\
& =2^{2 d} D_{h}^{2}(-1)^{d} \bar{g}(0) .
\end{aligned}
$$

It follows that

$$
\sqrt{D_{\bar{g}}}= \pm 2^{d} D_{h} \sqrt{(-1)^{d} \bar{g}(0)}
$$

Noting that $D_{h} \in K$ we have proved the following result.

Proposition 9. $K\left(\sqrt{D_{\bar{g}}}\right)=K\left(\sqrt{(-1)^{d} \bar{g}(0)}\right)$, whered $=\frac{1}{2}\left|G^{*}\right|=\frac{1}{2} \operatorname{deg}(\bar{g}(x))$. 
Proposition 10. Assume Condition Set holds. If $\bar{g}(x)$ is an irreducible factor of $g(x)$ over $K$, then the field extension $K\left(\sqrt{D_{\bar{g}}}\right)=K\left(\sqrt{(-1)^{d} \bar{g}(0)}\right)$ over $K$ has degree 2.

Proof. It suffices to show that $G^{*}$, viewed as a permutation group on the roots of $\bar{g}(x)$, contains an odd permutation. Fix a root $\xi$ of $\bar{g}(x)$. Then the map $\sigma \in G^{*} \mapsto \sigma \xi$ is a one-to-one correspondence from $G^{*}$ onto the set of roots of $\bar{g}(x)$, by Proposition 7. Thus we just need an element of $G^{*}$ acting as an odd permutation when $G^{*}$ acts on itself by left multiplication. Let $\rho$ be an element of $H^{*}$ of order $2^{m}$ and $\mu$ be an element of $H^{*}$ of order $h_{1}$. Then $H^{*}$ is the direct product of the two cyclic subgroups generated by $\rho$ and $\mu$ respectively. We also notice that $G^{*}=N H^{*}=H^{*} N$ since $N$ is a normal subgroup of $G^{*}$. Thus each element in $G^{*}$ can be represented uniquely as $\rho^{i} \mu^{j} \nu$ for some $\nu \in N, i \in\left\{0,1, \ldots, 2^{m}-1\right\}$ and $j \in\left\{0,1, \ldots, h_{1}-1\right\}$. We now claim that left multiplication by $\rho$, denoted $\rho_{L}: \sigma \in G^{*} \mapsto$ $\rho \sigma \in G^{*}$, serves as an odd permutation on the set $G^{*}$. For fixed $j \in$ $\left\{0,1, \ldots, h_{1}-1\right\}$ and $\nu \in N$, the action of $\rho_{L}$ is $\rho^{i} \mu^{j} \nu \mapsto \rho^{i+1} \mu^{j} \nu$ for $i \in\left\{0,1, \ldots, 2^{m}-2\right\}$ and $\rho^{2^{m}-1} \mu^{j} \nu \mapsto \mu^{j} \nu$. Therefore the cycle of length $2^{m}$

$$
\pi_{j, \nu}=\left(\mu^{j} \nu, \rho \mu^{j} \nu, \rho^{2} \mu^{j} \nu, \ldots, \rho^{2^{m}-1} \mu^{j} \nu\right)
$$

occurs in the representation of $\rho_{L}$ as the product of disjoint cycles, and

$$
\rho_{L}=\prod_{\substack{j=0 \\ \nu \in N}}^{h_{1}-1} \pi_{j, \nu} .
$$

As each $\pi_{j, \nu}$ is an odd permutation and $h_{1} n$ is an odd integer, $\rho_{L}$ is an odd permutation on $G^{*}$.

\section{PROOF OF THE THEOREM}

We verify that for all $i \in\{0,1, \ldots, t-1\}, K_{i}=K$ satisfies all five conditions in the Condition Set.

$f(x)$ is irreducible over $K_{0}=\mathbb{Q}$ by assumption. To show that $f(x)$ is irreducible over $K_{i}, i \in\{1,2, \ldots, t-1\}$, it suffices to show that the Galois group $\operatorname{Gal}\left(L / K_{i}\right)$ acts transitively on the set of roots of $f(x)$. But $\mathrm{Gal}\left(L / K_{i}\right)$ is, by Proposition $5, M_{i}=N \rtimes\left\langle\alpha^{2^{i}}\right\rangle$, which acts on $\left\{\theta_{1}, \ldots, \theta_{n}\right\}$ transitively by Proposition 4(iii). Hence (i) of the Condition Set holds.

It is clear that

$$
g(x)=\prod_{\substack{i, j=1 \\ i \neq j}}^{n}\left(x-\left(\theta_{i}-\theta_{j}\right)\right)
$$


is squarefree over $K_{i}$, and $L=K\left(\theta_{1}, \theta_{2}, \ldots, \theta_{n}\right)$ is the splitting field of $f(x)$. Thus (ii) and (iii) of the Condition Set hold.

The Galois group $\operatorname{Gal}\left(L / K_{i}\right)=M_{i}=N \rtimes\left\langle\alpha^{2^{i}}\right\rangle$ is a Frobenius group with kernel $N$ and complement $\left\langle\alpha^{2^{i}}\right\rangle$, which is a cyclic group of even order $2^{t-i} h_{1}$, where $t-i$ is a positive integer and $h_{1}$ is an odd positive integer. This verifies (iv) of the Condition Set. Finally, the degree of $f(x)$ is $n=|N|$ by assumption. Thus (v) of the Condition Set is valid.

Recall that the degree of $g(x)$ is $n(n-1)$. According to Proposition 7 and its corollary, each irreducible factor of $g(x)$ over $K_{i}$ is of degree $\left|G^{*}\right|=2^{t-i} h_{1} n=n h / 2^{i}$. Therefore $g(x)$ has $n(n-1) /\left|G^{*}\right|=2^{i}(n-1) / h$ irreducible factors over $K_{i}$. Hence over $K_{i}$ we have

$$
g(x)=\prod_{j=1}^{2^{i}(n-1) / h} g_{i j}(x),
$$

where each $g_{i j}(x) \in K_{i}[x]$ is monic, irreducible, and of degree $\left|G^{*}\right|=2^{t-i} h_{1} n=n h / 2^{i}$. By Proposition 10, the field extension $K_{i}\left(\sqrt{(-1)^{d_{i}} g_{i j}(0)}\right) / K_{i}$ has degree 2, where $d_{i}=\operatorname{deg}\left(g_{i j}(x)\right) / 2$. It is now clear that for $i \in\{0,1, \ldots, t-1\}$, the degree of the element $\sqrt{(-1)^{d_{i}} g_{i j}(0)}$ over the rational field $\mathbb{Q}$ is $2^{i+1}$. By the uniqueness of the quadratic tower (11) (Remark 2), we have

$$
K_{i+1}=\mathbb{Q}\left(\sqrt{(-1)^{d_{i}} g_{i j}(0)}\right), \quad i \in\{0,1, \ldots, t-1\} .
$$

When $i \in\{0, \ldots, t-2\}, d_{i}=\operatorname{deg}\left(g_{i j}(x)\right) / 2=2^{t-1-i} h_{1} n$ is even, and it follows that $\sqrt{(-1)^{d_{i}} g_{i j}(0)}=\sqrt{g_{i j}(0)}$.

When $i=t-1, d_{t-1}=\operatorname{deg}\left(g_{t-1 j}(x)\right) / 2=2^{t-1-(t-1)} h_{1} n=h_{1} n$ is odd, hence we have $\sqrt{(-1)^{d_{t-1}} g_{t-1 j}(0)}=\sqrt{-g_{t-1 j}(0)}$.

The proof is now complete since both (13) and (14) are established by (15) and the notes above.

\section{EXAMPLES}

Our theorem gives a practical way of determining the normal subfields $K_{i}$ of degree $2^{i}$ of the splitting field of $L$ of $f$ since the polynomial $g(x)$ can be conveniently computed using resultants (see Soicher, 1981) and factored over a number field using for example a package such as 
MAPLE. If $g(x)$ has repeated factors it is necessary to change the polynomial $f(x)$ by a Tschirnhausen transformation.

Example 1. Let $f(x)=x^{5}-5 a x^{3}+5 a^{2} x-b \in \mathbb{Z}[x]$ be irreducible. Then $4 a^{5}-b^{2} \neq 0$, otherwise there exists an integer $c$ such that $a=c^{2}, b=2 c^{5}$, and $f(x)$ has the linear factor $x-2 c$. The Galois group $G$ of $f$ is the Frobenius group $F_{20}$. Here $n=5, h=4,(n-1) / h=1$ and $t=2$. The polynomial $f(x)$ is known as DeMoivre's quintic. Set

$$
g(x)=\frac{\operatorname{Resultant}(f(x+X), f(X))}{x^{5}} .
$$

MAPLE gives $g(x)$ as a polynomial of degree 20 with constant term $g(0)=5^{5}\left(4 a^{5}-b^{2}\right)^{2}=g_{01}(0)$. By our theorem the unique quadratic subfield $K_{1}$ of $L$ is

$$
K_{1}=\mathbb{Q}(\sqrt{g(0)})=\mathbb{Q}(\sqrt{5}) .
$$

Next we factor $g(x)$ in $\mathbb{Q}(\sqrt{5})[x]$. MAPLE gives two monic polynomials $g_{11}(x)$ and $g_{12}(x)$ in $\mathbb{Q}(\sqrt{5})[x]$ of degree 10 such that

$$
g(x)=g_{11}(x) g_{12}(x) .
$$

By our theorem these polynomials are irreducible in $\mathbb{Q}(\sqrt{5})[x]$. Evaluating them at $x=0$, MAPLE gives

$$
g_{11}(0)=\frac{1000 a^{5}-250 b^{2}}{-25+11 \sqrt{5}}, \quad g_{12}(0)=\frac{1000 a^{5}-250 b^{2}}{-25-11 \sqrt{5}},
$$

and our theorem yields the unique quartic subfield $K_{2}$ of $L$ as

$$
K_{2}=\mathbb{Q}\left(\sqrt{-\left(\frac{1000 a^{5}-250 b^{2}}{-25+11 \sqrt{5}}\right)}\right) .
$$

Since

$$
-\left(\frac{1000 a^{5}-250 b^{2}}{-25+11 \sqrt{5}}\right)=\left(\frac{5+5 \sqrt{5}}{2}\right)^{2}\left(4 a^{5}-b^{2}\right)(5+2 \sqrt{5}),
$$

we have

$$
K_{2}=\mathbb{Q}\left(\sqrt{\left(4 a^{5}-b^{2}\right)(5+2 \sqrt{5})}\right)
$$

in agreement with Spearman and Williams (1999, Theorem). 
Example 2. We choose

$$
f(x)=x^{5}+a x+b \in \mathbb{Z}[x]
$$

to be a solvable, irreducible quintic trinomial with $a b \neq 0$. Let $r$ be the unique rational root of the resolvent sextic of $x^{5}+a x+b$ (Spearman and Williams, 1994, p. 988). Set

$$
c=\left|\frac{3 r-16 a}{4 r+12 a}\right|, \quad \varepsilon=\operatorname{sgn}\left(\frac{3 r-16 a}{4 r+12 a}\right), \quad e=\frac{-5 b \varepsilon}{2 r+4 a},
$$

so that

$$
c(\geq 0) \in \mathbb{Q}, \quad \varepsilon= \pm 1, \quad e(\neq 0) \in \mathbb{Q} .
$$

Then (see, for example, Spearman and Williams, 1994, Theorem, p. 987) we have

$$
a=\frac{5 e^{4}(3-4 \varepsilon c)}{c^{2}+1}, \quad b=\frac{-4 e^{5}(11 \varepsilon+2 c)}{c^{2}+1} .
$$

The Galois group $G$ of $f$ is

$$
\left\{\begin{array}{cc}
D_{5}, & \text { if } 5\left(c^{2}+1\right) \in \mathbb{Q}^{2}, \\
F_{20}, & \text { if } 5\left(c^{2}+1\right) \notin \mathbb{Q}^{2},
\end{array}\right.
$$

where $D_{5}$ is the dihedral group of order 10 and $F_{20}$ is the Frobenius group of order 20 (Spearman and Williams, 1994, p. 990). We note that $D_{5}$ is a Frobenius group. We just treat the case when $G=F_{20}$ as the case $G=D_{5}$ is simpler. Here $n=5, h=4,(n-1) / h=1$ and $t=2$. Set

$$
g(x)=\frac{\operatorname{Resultant}(f(x+X), f(X))}{x^{5}} .
$$

MAPLE gives $g(x)$ as a polynomial of degree 20 with constant term

$$
g(0)=g_{01}(0)=2^{8} 5^{5} \frac{\left(4 \varepsilon c^{3}-84 c^{2}-37 \varepsilon c-122\right)^{2}}{\left(c^{2}+1\right)^{5}} .
$$

By the theorem we obtain

$$
K_{1}=\mathbb{Q}\left(\sqrt{g_{01}(0)}\right)=\mathbb{Q}\left(\sqrt{5\left(c^{2}+1\right)}\right),
$$

in agreement with Spearman et al. (1995, p. 16).

Next we use MAPLE to factor $g(x)$ over $K_{1}$. MAPLE gives $g(x)$ as

the product of two monic polynomials $g_{11}(x)$ and $g_{12}(x)$ 
in $\mathbb{Q}\left(\sqrt{5\left(c^{2}+1\right)}\right)[x]$ of degree 10 such that

$$
g(x)=g_{11}(x) g_{12}(x) .
$$

MAPLE gives

$$
\begin{aligned}
g_{11}(0) & =(\text { square }) \times\left(-25\left(c^{2}+1\right)+(5+10 \varepsilon) \sqrt{5\left(c^{2}+1\right)}\right) \\
& =(\text { square }) \times 5\left(c^{2}+1\right)\left(-5+(1+2 \varepsilon) \sqrt{\frac{5}{c^{2}+1}}\right) .
\end{aligned}
$$

By the theorem we have

$$
K_{2}=\mathbb{Q}\left(\sqrt{-5+(1+2 \varepsilon) \sqrt{\frac{5}{c^{2}+1}}}\right)
$$

in agreement with Spearman et al. (1995, Theorem, p. 17).

We conclude by giving brief details of four numerical examples.

\section{Example 3.}

$$
\begin{aligned}
f(x) & =x^{5}-70 x^{3}-140 x^{2}+385 x+28, \\
G & =F_{20}, n=5, h=4,(n-1) / h=1, t=2, \\
g_{01}(0) & =2^{17} 5^{5} 7^{4} 43^{2}, \\
K_{1} & =\mathbb{Q}(\sqrt{10}), \\
g_{11}(0) & =2^{8} 5^{2} 7^{2}(-650+201 \sqrt{10}), \\
g_{12}(0) & =2^{8} 5^{2} 7^{2}(-650-201 \sqrt{10}), \\
K_{2} & =\mathbb{Q}(\sqrt{650+201 \sqrt{10}}) \\
& =\mathbb{Q}\left(\sqrt{\left(\frac{17+4 \sqrt{10}}{3}\right)^{2}(10+\sqrt{10})}\right) \\
& =\mathbb{Q}(\sqrt{10+\sqrt{10}}) .
\end{aligned}
$$




\section{Example 4.}

$$
\begin{aligned}
f(x)= & x^{9}-3 x^{8}+3 x^{7}-15 x^{6}+33 x^{5}-3 x^{4}+24 x^{3}+6 x^{2}-4, \\
& \text { see }(\text { Cangelmi, 2000, p. 856), } \\
= & \left(\mathbb{Z}_{3} \times \mathbb{Z}_{3}\right) \rtimes \mathbb{Z}_{4}, \\
n= & 9, h=4,(n-1) / h=2, t=2, \\
g_{01}(0)= & 2^{8} 3^{6} 5^{7}, \\
g_{02}(0)= & 2^{4} 3^{6} 5^{11} \\
K_{1}= & \mathbb{Q}(\sqrt{5}) \\
g_{11}(0)= & 2^{4} 3^{3} 5^{3}(5+2 \sqrt{5}), \\
g_{12}(0)= & 2^{4} 3^{3} 5^{3}(5-2 \sqrt{5}), \\
g_{13}(0)= & 23^{3} 5^{5}(5-\sqrt{5}), \\
g_{14}(0)= & 23^{3} 5^{5}(5+\sqrt{5}), \\
K_{2}= & \mathbb{Q}(\sqrt{-(15+6 \sqrt{5})}) \\
= & \mathbb{Q}\left(\sqrt{-\left(\frac{1+\sqrt{5}}{4}\right)^{2}(30+6 \sqrt{5})}\right) \\
= & \mathbb{Q}(\sqrt{-(30+6 \sqrt{5})}) .
\end{aligned}
$$

\section{Example 5.}

$$
\begin{aligned}
f(x)= & x^{9}-72 x^{7}+1464 x^{5}-960 x^{4}-8928 x^{3}+13440 x^{2} \\
& -2064 x-2560 .
\end{aligned}
$$

The MAGMA database gives

$$
G=T_{15} \text { (notation of Butler and McKay, 1983), }|G|=72 .
$$

The group $T_{15}$ has one normal subgroup $N=\mathbb{Z}_{3} \times \mathbb{Z}_{3}$ of order 9 as well as nine conjugate subgroups of order 8 , each of which is cyclic. These conjugate subgroups intersect only trivially so $G$ is a Frobenius group and is the semidirect product $\left(\mathbb{Z}_{3} \times \mathbb{Z}_{3}\right) \rtimes \mathbb{Z}_{8}$. 


$$
\begin{aligned}
n= & 9, h=8,(n-1) / h=1, t=3, \\
g_{01}(0)= & 2^{67} 3^{12} 5^{6} 7^{2} 239^{2} 503^{2}, \\
K_{1}= & \mathbb{Q}(\sqrt{2}), \\
g_{11}(0)= & 2^{33} 3^{6} 5^{3}(2 \cdot 29 \cdot 137 \cdot 1193+6650041 \sqrt{2}), \\
K_{2}= & \mathbb{Q}(\sqrt{2 \cdot 5 \cdot 29 \cdot 137 \cdot 1193+5 \cdot 6650041 \sqrt{2}}) \\
= & \mathbb{Q}(\sqrt{10-5 \sqrt{2}}), \\
g_{21}(0)= & -2^{16} 3^{2}\left(5662200+3307230 \beta-330870 \beta^{2}-193803 \beta^{3}\right), \\
& \text { where } \beta=\sqrt{10-5 \sqrt{2}}, \\
K_{3}= & \mathbb{Q}\left(\sqrt{5662200+3307230 \beta-330870 \beta^{2}-193803 \beta^{3}}\right) .
\end{aligned}
$$

Since

$$
\begin{aligned}
& \left(5662200+3307230 \beta-330870 \beta^{2}-193803 \beta^{3}\right)\left(30-3 \beta+\frac{3 \beta^{2}}{5}\right) \\
& \quad=\left(9450+5820 \beta-531 \beta^{2}-336 \beta^{3}\right)^{2}
\end{aligned}
$$

we have

$$
\begin{aligned}
K_{3} & =\mathbb{Q}\left(\sqrt{30-3 \beta+\frac{3 \beta^{2}}{5}}\right) \\
& =\mathbb{Q}(\sqrt{30-3 \sqrt{10+5 \sqrt{2}}+6 \sqrt{10-5 \sqrt{2}}}) .
\end{aligned}
$$

\section{Example 6.}

$$
\begin{aligned}
f(x)= & x^{13}-26 x^{10}-117 x^{8}+143 x^{7}-910 x^{6}+585 x^{5} \\
& -1794 x^{4}+4472 x^{3}-2951 x^{2}+520 x-131 .
\end{aligned}
$$

MAPLE gives the discriminant of $f(x)$ as $2^{8} 13^{21} 43^{2} 2791^{2}$ $332699^{2} 15515891^{2}$ so that the quadratic subfield of $L$ is $\mathbb{Q}(\sqrt{13})$. If $\alpha$ is 
any root of $f(x)$ MAPLE factors $f(x)$ over $\mathbb{Q}(\alpha, \sqrt{13})$. There are six irreducible quadratics and one linear polynomial in the factorization. Hence

$$
[L: \mathbb{Q}]=2^{k} 13
$$

for some $k \in \mathbb{Z}^{+}$. Therefore $f(x)$ is solvable so $\operatorname{Gal}(f)=F_{13 l}$, where $l \mid 12$. It is known that $[L: \mathbb{Q}]=13 l$, where $l \neq 1$ as $L$ has a quadratic subfield and $l \neq 2$ as $f$ does not factor into linear factors over $\mathbb{Q}(\alpha, \sqrt{13})$. Hence $l=4$ and $\operatorname{Gal}(f)=F_{52}$. We remark that a theorem of Cangelmi (2000, Theorem 3.17, p. 851) provides an alternative way of verifying that $\operatorname{Gal}(f)=F_{52}$.

$$
\begin{aligned}
n & =13, h=4,(n-1) / h=3, t=2, \\
g_{01}(0) & =13^{7} 15515891^{2}, \\
g_{02}(0) & =2^{8} 13^{7} 332699^{2}, \\
g_{03}(0) & =13^{7} 43^{2} 2791^{2}, \\
K_{1} & =\mathbb{Q}(\sqrt{13}), \\
g_{11}(0) & =\frac{13^{3}}{2}\left(5 \cdot 13^{2} \cdot 1822217+3^{3} \cdot 3793 \cdot 4159 \sqrt{13}\right), \\
K_{2} & =\mathbb{Q}\left(\sqrt{-\frac{1}{2}\left(5 \cdot 13^{2} \cdot 1822217+3^{3} \cdot 3793 \cdot 4159 \sqrt{13}\right.}\right) \\
& =\mathbb{Q}(\sqrt{-13-2 \sqrt{13}}) .
\end{aligned}
$$

\section{ACKNOWLEDGMENTS}

The authors are grateful to the referee for his/her invaluable suggestions based on his/her thorough reading and understanding of our work. The research of the first two authors was supported by grants from the Natural Sciences and Engineering Research Council of Canada.

\section{REFERENCES}

Butler, G., McKay, J. (1983). The transitive groups of degree up to eleven. Comm. Algebra 11:863-911.

Cangelmi, L. (2000). Polynomials with Frobenius Galois groups. Comm. Algebra 28:845-859. 
Robinson, D. J. S. (1982). A Course in the Theory of Groups. Graduate Text in Mathematics 80. New York: Springer-Verlag.

Rotman, J. (2002). Advanced Modern Algebra. Prentice-Hall.

Soicher, L. (1981). M. Comp. Sci. thesis, Concordia University, Montréal.

Spearman, B. K., Williams, K. S. (1994). Characterization of solvable quintics $x^{5}+a x+b$. Amer. Math. Monthly 101:986-992.

Spearman, B. K., Williams, K. S. (1999). DeMoivre's quintic and a theorem of Galois. Far East J. Math. Sci. (FJMS) 1:137-143.

Spearman, B. K., Spearman, L. Y., Williams, K. S. (1995). The subfields of the splitting field of a solvable quintic trinomial. J. Math. Sci. 6:15-18.

Received November 2001

Revised December 2002 